\title{
CURE CONTROL: STRATEGIES FOR USING DIELECTRIC SENSORS
}

David R. Day

Micromet Instruments, Inc.

21 Erie Street, Cambridge, MA 02139

\section{INTRODUCTION}

Dielectric measurements are becoming increasingly important as a means for feedback control in the area of polymer processing. The changes in dielectric response as a function of changing molecular weight or cross link density have been the subject of much research for the last 50 years [1]. Only now is process control through dielectric feedback becoming a reality due to recent advances in dielectric measurement capability [2]. Microdielectric sensors are now available which function down to frequencies characteristic of mechanical measurements (less than $1 \mathrm{~Hz}$ ) and can be inserted directly into curing composite structures. By monitoring the dielectric properties (permittivity and loss factor) at several frequencies, the ionic conductivity can be extracted [3] with the aid of commercial software packages in real time. This paper investigates the use of first and second derivative information (log ionic conductivity with respect to time) for controlling certain key processining steps during composite curing.

\section{EXPERIMENTAL}

Dielectric analysis was carried out with a Micromet Instruments Eumetric System II Microdielectrometer. Microdielectric sensors were used to monitor the dielectric response over the frequency range of 0.005 to $10,000 \mathrm{~Hz}$. Materials monitored include a Fiberite 934 graphite epoxy, a USP graphite PMR-15, and a Hexcell 8-297 graphite epoxy. In each case a small piece of glass felt was placed over the sensor to prevent the graphite from coming into contact with the sensor. Temperature was controlled with a Micromet Eumetric Programmable Oven and system software. The commercially available dielectric monitoring software was modified to calculate the derivative of ionic conductivity with respect to time in real time. When the programmed conditions were satisfied, the computer in the first two examples drew shaded areas on the real time plot simulating the initiation of a process control step. This modified software will subsequently be referred to as the Process Control Software (PCS). In the third example, the computer actually controlled the temperature of the oven based on the microdielectric sensor 


\section{Detecting Viscosity Minima}

Figure 1 shows a typical industrial cure cycle for a graphite epoxy prepreg, in this case the Hexcell 8-297 material. The left axis of this plot, log conductivity, was extracted from multifrequency loss factor data. The quantity of $\log$ conductivity axis may be thought of as a fluidity axis or log (1/viscosity). The peaks in Figure 1 therefore represent fluidity maxima or viscosity minima. It is often the case that pressure is applied near the second viscosity minimum to insure good flow but not too much bleed. In order to trigger pressure application at the second fluidity maximum the software must be able to detect a zero slope of the log conductivity as it passes through a maximum.

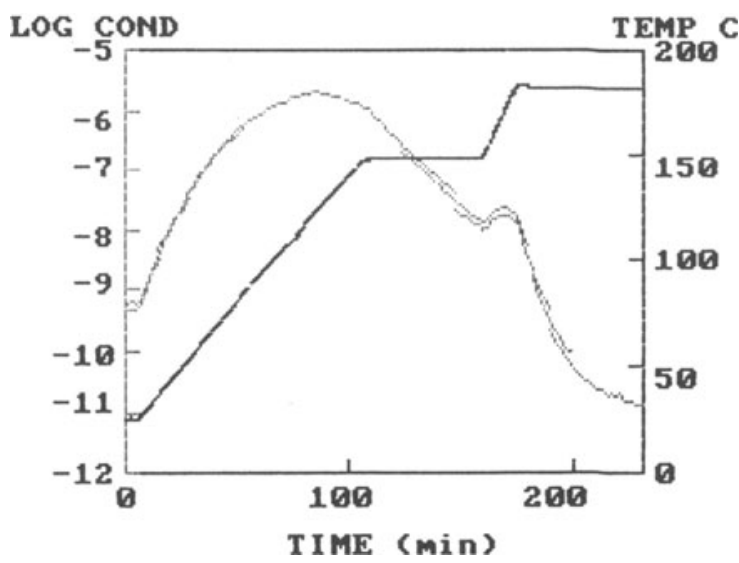

Figure 1. Log conductivity (extracted from 1 to $10,000 \mathrm{~Hz}$ loss factor data, during cure of Hexcell 8-927 graphite epoxy.

In order to set this triggering action the PCS software has the parameter menu shown in Figure 2. The first parameters required are the time and temperature window. The first number represents a value below which the process will not be triggered. For example, if the slope is 0 but the temperature has not yet reached $150 \mathrm{C}$, the process will not be triggered. This prevents the software, for example, from applying pressure at the first fluidity maximum in figure 1 . The second number in the time and temperature window is a value that if attained will automatically trigger the process (if it hasn't been already) no matter what the slope is.

The next parameter is "critical slope". This represents the value of the derivative of $\log$ conductivity with respect to time at which the process should be triggered. This is related to the "trend" parameter which defines whether the process trigger should occur if the slope is passing in a positive or negative direction through the critical slope. 
The "trend" parameter represents what the sign of the second derivative should be when the critical slope is attained. For a fluidity maxima the "trend" parameter should be set to negative since the slope will be going from positive to negative as it passes through zero.
A. TIME WINDOW $=150300$
B. TEMP WINDOW= 150200
C. CRIT. SLOPE $=0$
D. ON POS OR MEG TREMD=NEGATIUE
E. \# OF CYCLES TO AUE. $=2$

Figure 2. Process Control Software parameter menu.

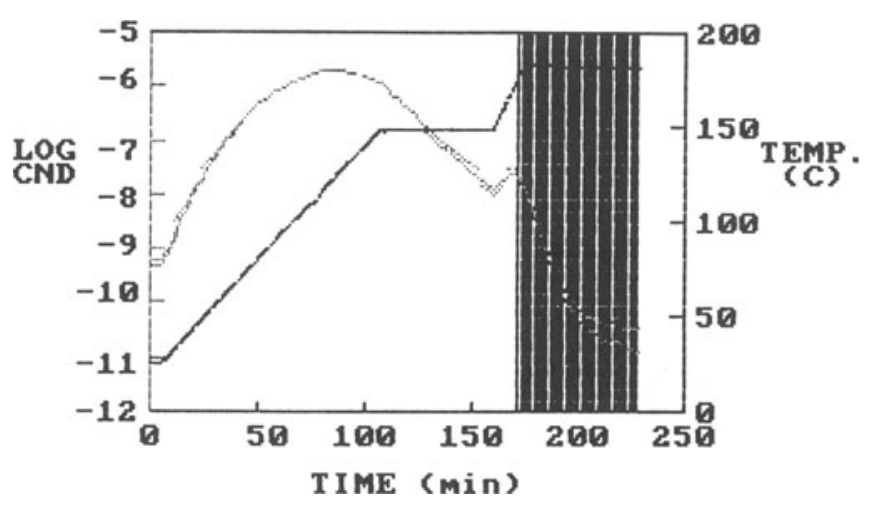

Figure 3. Graphite epoxy cure (from Fig. 1) using control parameters (from figure 2). Shaded area is where software applies pressure

The final parameter is the number of data sets (n) to average in determining the slope. The PCS takes all the ionic conductivity data from $(n+1)$ frequency cycles and does a least squares fit to determine slope. It has been found that mid-process trigger point detection only requires $n$ to be 1 or 2 for reliable performance. End of cure detection may require more depending on how small a slope is required.

The shaded area in Figure 3 shows where pressure is applied based on the PCS parameters used in Figure 2. Note that pressure is applied almost exactly as the second fluidity maximum is attained. 


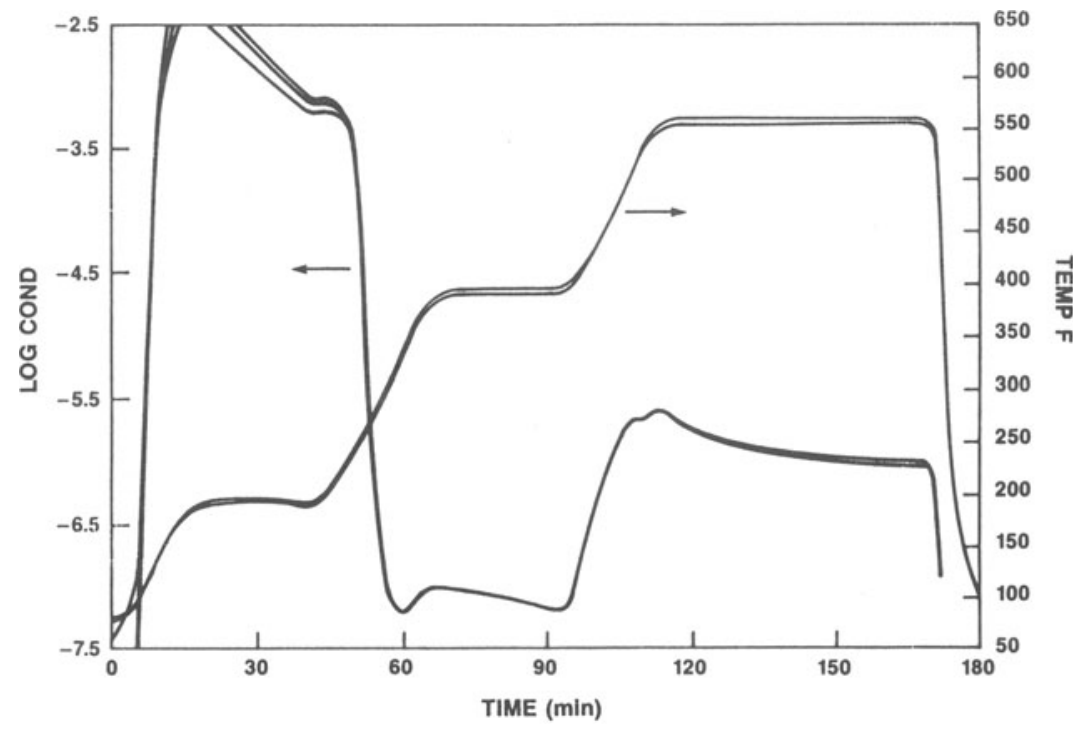

Figure 4. Log conductivity (extracted from 1 to $1000 \mathrm{~Hz}$ loss factor data) during cure of USP PMR-15 graphite polyimide.

\section{Detecting Onset of Reaction}

Pressure application is again a critical point during the cure of PMR-15, a graphite polyimide material. The ionic conductivity extracted from mulitfrequency loss factor data during a standard PMR-15 cure is shown in Figure 4. The first large fall off $\log$ conductivity is due to imidization along the polymer backbone. During the final ramp up to $600 \mathrm{~F}$ the chain ends undergo a second crosslinking reaction. This corresponds to the point at about 110 minutes where the ionic conduction bends over with a slightly lower slope. This is precisely where it is desirable to apply pressure (before crosslinking prohibits flow). Although the change in ionic conduction level is minor at this critical point, the change in the slope with respect to time is considerable.

Figure 5 shows the first derivative of ionic conduction with respect to time from Figure 4. The value of the first derivative falls from a value of about 1.1 to lower values at the onset of crosslinking. It is precisely at this point that pressure triggering should occur. In order to set the trigger point appropriately, the PCS parameters are set as follows. The temperature window is set at 450,600 preventing any pressure application before $450 \mathrm{~F}$ is attained. The time window is set to 0,1000 (not a critical parameter). The critical slope is set to .08 so the pressure application will occur just as the slope starts to fall from the .13 value. The "trend" parameter is set to negative to insure that triggering occurs as the slope is decreasing (and not during the increase around 95 minutes). Finally the number of point averaged is set to 2 .

Figure 6 shows the results utilizing these parameters. The shaded area represents where pressure is applied. Note that as the second temperature ramp was initiated the ionic conductivity level started to increase, due to a steady increase in ionic mobility. However, as the crosslinking starts to occur, the rate of increase if ion mobility, hence ionic conductivity, is impeded and the slope starts to decrease slightly. This PCS detected the decrease and triggered the pressure at exactly this point. 


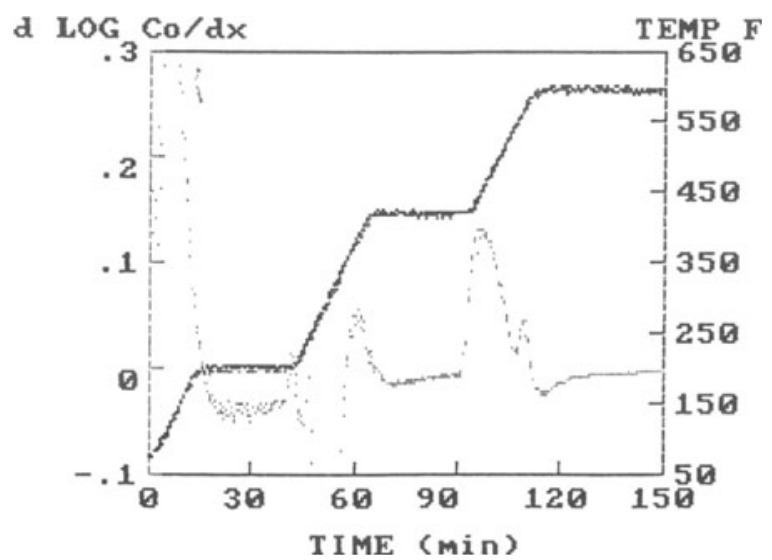

Figure 5. Slope of Log conductivity (from Fig.4) during cure.

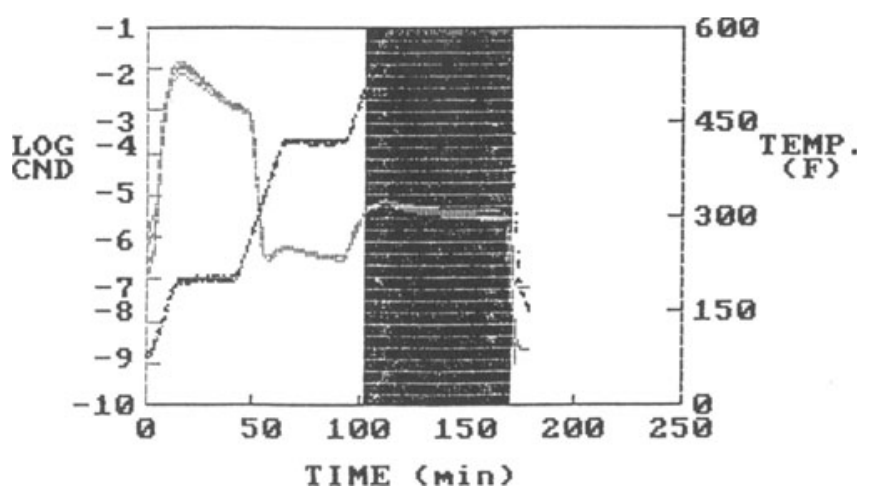

Figure 6. PMR-15 cure using control parameters adjusted to trigger pressure application at onset of crosslinking reaction. Shaded area represents application of pressure. 
The previous cure control examples were carried out using PCS, a routine intended for generic process application. The next example was carried out by a specialized software routine written specifically for the cure Fiberite 934 graphite epoxy. The program is composed of five main subroutines and was designed to monitor dielectric and temperature information from the microdielectric sensor and automatically control the oven temperature. In Figure 7, the ionic conductivity is converted to resistivity (by inverting) and is labelled as "ion viscosity" (due to the close relation between resistivity and actual viscosity and rigidity). When the condition for each subroutine is met, the program automatically jumps to the next routine. The five subroutines are listed below:

1. heat material to $250 \mathrm{~F}$

2. hold at $250 \mathrm{~F}$ until ion viscosity reaches 7.0

3. hold ion viscosity at 7.0 (by controlling temperature) until temperature reaches $350 \mathrm{~F}$

4. hold at $350 \mathrm{~F}$ until slope of ion viscosity with respect to time is zero

5. cool down and notify operator

Note that after heat up to $250 \mathrm{~F}$ the computer waits for the ion viscosity to increase to 7 . Control step 3 is perhaps the most interes-ing. During this segment, the computer notices small increases in ion viscosity and automatically increases the temperature to drive in the ion viscosity back to 7 . The resulting profile during this period is curved and sucessfully eliminates the second viscosity minimum. In standard cure cycles, a linear temperature ramp is used, thus causing a second viscosity minimum to occur. Note that after a period of time the temperature can not be increased fast enough and the ion viscosity starts to build rapidly. The program then holds the temperature at $350 \mathrm{~F}$ until the change in ion viscosity with respect to time reaches zero.

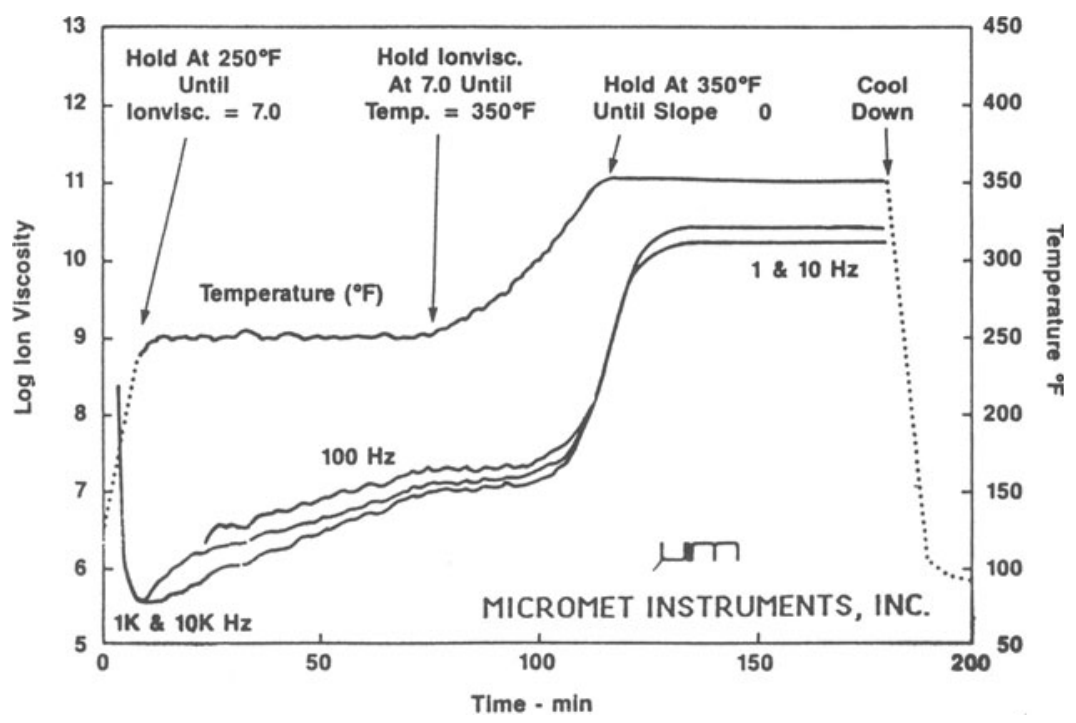

Figure 7. Closed loop cure of Fiberite epoxy graphite material. Control routine are noted in figure. 


\section{CONCLUSIONS}

Microdielectric sensors in combination with the developed software are capable of performing closed loop processing using a variety of control options. The Process Control Software may be instructed to trigger a process step through monitoring of slope of extracted ionic conductivity with respect to time. The additional user selected parameters of time and temperature window, trend, and number of points to average, enable the Process Control Software to very specifically pinpoint the appropriate process trigger point.

More sophisticated control is possible in which the entire cure process may be "intelligently" directed. Hold times may be based on reaching critical values of ionic conduction or slope of conduction with respect to time and viscosity may be controlled in real time by continuously changing temperature.

\section{REFERENCES}

1. R. Kienle and H. Race, Trans. Electrochem. Soc., 65, 87 (1934)

2. S.D. Senturia, N.F. Sheppard Jr., H.L. Lee, S.B. Marshall, SAMPE J., 19, 22 (1983)

3. D.R. Day, . Eng. Sci., 26, 5, 362 (1986) 